\title{
Application of Monte Carlo Chord-Length Sampling Algorithms to Transport Through a 2-D Binary Stochastic Mixture
}

\author{
T.J. Donovan, Y. Danon
}

This report was prepared as an account of work sponsored by the United States Government. Neither the United States, nor the United States Department of Energy, nor any of their employees, nor any of their contractors, subcontractors, or their employees, makes any warranty, express or implied, or assumes any legal liability or responsibility for the accuracy, completeness or usefulness of any information, apparatus, product or process disclosed, or represents that its use would not infringe privately owned rights. 


\title{
Application of Monte Carlo Chord-Length Sampling Algorithms to Transport Through a 2-D Binary Stochastic Mixture
}

\author{
T. J. Donovan', Y. Danon \\ Lockheed Martin Corp, Schenectady, NY \\ Nuclear Engineering Department, Rensselaer Polytechnic Institute, Troy, NY
}

ABSTRACT

\begin{abstract}
Monte Carlo algorithms are developed to calculate the ensemble-average particle leakage through the boundaries of a 2-D binary stochastic material. The mixture is specified within a rectangular area and consists of a fixed number of disks of constant radius randomly embedded in a matrix material. The algorithms are extensions of the proposal of Zimmerman et al., using chord-length sampling to eliminate the need to explicitiy model the geometry of the mixture. Two variations are considered. The first algorithm uses Chord-Length Sampling (CLS) for both material regions. The second algorithm employs Limited Chord Length Sampling (LCLS), only using chord-length sampling in the matrix material. Ensemble-average leakage results are computed for a range of material interaction coefficients and compared against benchmark results for both accuracy and efficiency. Both algorithms are exact for purely absorbing materials and provide decreasing accuracy as scattering is increased in the matrix material. The LCLS algorithm shows a better accuracy than the CLS algorithm for all cases while maintaining an equivalent or better efficiency. Accuracy and efficiency problems with the CLS algorithm are due principally to assumptions made in determining the chord-length distribution within the disks.
\end{abstract}

\section{Introduction}

Interest has continued to grow in recent years regarding methods that solve the particle transport equation for systems whose material properties are only known in a statistical sense $(1-16)$. These stochastic mixture systems by definition contain two or more immiscible materials arranged randomly. The special case of two immiscible materials in a random arrangement is referred to as a binary stochastic mixture. The binary stochastic mixture has received particular attention in the literature due to its relative simplicity and applicability to a number of physical problems. These applications include stochastic stellar or atmospheric media $(1,2,3)$, heterogeneous shield material (4), two-phase coolant, or fractured geological material (5).

The statistical nature of the geometry of a stochastic mixture adds a significant complication to the solution of the underlying transport equation. Because the material properties are at best only known statistically it is not possible to explicitly model the material properties of the problem. The transport equation in its traditional form therefore cannot be directly applied to a stochastic mixture. Because the material properties are not known explicitly, the particle flux as a function of position is a spatially random quantity that is very difficult to compute deterministically. . Exact analytical solutions for transport in a binary stochastic medium can only be derived for simple cases (6) and are of limited usefulness. The ideal approach to the stochastic mixture problem would be to find a method to determine the probability distribution function of the flux $\psi(\mathrm{r})$. However, because this approach is rarely feasible most methods are concerned with determining the ensemble-averaged flux. Some methods have also been developed to determine the second moment, or the variance (7) of the flux.

\footnotetext{
I To whom all correspondence should be addressed: donovat@kapl.gov
} 
A general stochastic transport model was developed by Adams et al (8). This model is valid for a binary stochastic mixture with Markovian mixing statistics. Markovian mixing statistics are defined by

$$
P(i \rightarrow j)=\frac{d s}{\lambda_{i}(s)}, j \neq i
$$

Where $P(i \rightarrow j)$ is the differential probability that, starting from material $\mathrm{i}$, point $\mathrm{s}+\mathrm{d}$ is in material $\mathrm{j} \neq \mathrm{i}$. The $\lambda_{i}(s)$ parameters are the Markovian transition lengths which completely define the statistics of the mixture. For homogeneous mixing statistics the transition length is independent of $s$ and can be interpreted as the mean chord-length through packets of material $\mathrm{i}$ and in direction $\Omega$. Although exact for the specified mixing statistics, the difficulty with the Reference (8) model is that it is not closed since it contains two equations and four unknowns. The additional two unknowns are interface ensemble-averaged flux contributions, conditional upon the position $\hat{r}$ being on the interface between material $i$ and material $j$. These additional terms can be interpreted as additional source terms in the stochastic transport equation accounting for particles leaving one material and entering the other. A number of efforts have been made to provide closure to this model. These efforts range in complexity from the simple and well-known Levermore approximation ( 8 ) to higher order closure schemes of varying complexity $(9,10,11,12)$. The accuracy of some of these closure methods was studied by Pomraning (13) who concluded, as expected, that accuracy is improved with increased model complexity.

The complexity of the coupled stochastic transport equation, especially when combined with higher order closure equations, makes this problem extremely challenging to solve. Most recent efforts have presented deterministic methods or hybrid deterministic/Monte Carlo methods for solving these types of problems. These hybrid methods use Monte Carlo only in a loose definition of the technique in that random sampling is only used to generate many individual realizations of the random mixture that are then solved using deterministic methods. Analog Monte Carlo techniques have mostly been applied only to compute so called "benchmark" results $(5,14,8)$. These benchmark results have then been used in providing comparisons to results obtained using these alternate methods.

Calculation of benchmark results for stochastic mixtures using Monte Carlo is a very costly exercise. The ensemble averaged transport solution is computed in the following way: 1) build a specific realization of the stochastic mixture by sampling from the given mixing statistics, 2) perform a Monte Carlo simulation for that specific realization and tally the quantities of interest, 3 ) repeat for a large number of realizations and average the results. This method is exact in the limit of probability as the number of realizations approached infinity. However, this method is too costly to be practical for routine use.

Zimmerman and Adams $(14,15)$ reasoned that the mixing statistics for a binary mixture could be incorporated into the Monte Carlo transport solution to provide an approximation of the ensemble averaged-solution. This is a natural extension of the Monte Carlo method, which is based on random sampling from known probability distributions. Zimmerman and Adams proposed three algorithms of 
increasing complexity based on chord-length sampling. These algorithms were used to compute leakage and reflection results for 1-D rod geometry problems. These results showed that the pure chord-length sampling method is equivalent to solving the Levermore equations. Furthermore, the retention of information about the sampled material distribution was shown to improve accuracy at the cost of increased computational complexity.

The reported early success of Monte Carlo chord-length sampling methods by Zimmerman and Adams for simple 1-D stochastic mixture problems warrants further investigation of the use and performance of these techniques in multidimensional problems. The extension of these techniques becomes more important considering the increasing dependence on Monte Carlo methods for tackling large multidimensional problems combined with the increasing computing power available to bring to bear on such problems. Given the wide list of applications that deal with stochastic mixtures, the ability to model stochastic mixtures within large scale models using a powerful standardized Monte Carlo code could find a considerable audience. Such a capability would eliminate the need to either model such mixtures as homogeneous, a simplification that is known to be of limited validity $(16,13)$, or spend the considerable effort to explicitly model the stochastic mixture.

The purpose of this work is to extend the concept of chord-length sampling into a specified 2-dimensional problem. A pure chord-length sampling technique, and the derivations of the appropriate chord-length distributions, is described for the problem. In addition, a second technique is developed which eliminates chord-length sampling in one of the materials. Ensemble-averaged results obtained with both techniques are then compared to benchmark results obtained using standard Monte Carlo. Results are also compared to results generated using a simple homogenization of the stochastic mixture in order to show that for the problems investigated, the homogenization technique does not produce acceptable results.

\section{Analysis}

\section{II.I Description of problem:}

Figure 1 represents the 2-D stochastic mixture to be analyzed. The bounding area for the problem is a rectangle of arbitrary dimensions. The particle source will be defined as originating outside of the bounding volume and incident on the left side. For simplicity, the source is defined to be a pencil beam normal to the rectangle wall. This problem is monoenergetic in order to simplify the analysis. However energy dependence in the source and material interaction coefficients could easily be included without altering the chord-length sampling algorithms which are of principal concern.

The binary stochastic mixture consists of disks of radius $r$ and material 0 surrounded by a matrix of material 1. Materials 0 and 1 possess both absorption and scattering coefficients. Scattering is treated as isotropic in the lab system, meaning that a particle's exiting direction, theta, is sampled uniformly from 0 to $2 \pi$. The interaction coefficients of both materials will be varied for different cases. The number of disks 
contained in the rectangle is fixed for all problems. Disks are randomly placed within the rectangle with the constraint that they may not overlap other disks or the rectangle boundaries.

The goal of the analysis is to calculate the mean leakage through each of the four walls of the bounded area. Leakage means the fraction of source particles that escape the system by passing through each of the four walls. The second goal of this analysis is to compare the computational efficiency of the two algorithms to that of the benchmark method. Since the absolute times will vary significantly between platforms, these results are normalized by the elapsed time required for the benchmark results in each case.

Six cases were selected to cover a range of interaction coefficients. Table 1 gives the materials' interaction coefficients for the six cases.

TABLE 1: Material Scatter and Absorption Coefficients for 6 Cases

\begin{tabular}{|c|c|c|c|c|}
\hline CASE \# & $\begin{array}{c}\text { Material } 0 \\
\text { Scatter } \\
\text { Coefficient } \\
\Sigma_{\mathrm{s}, 0}(1 / \mathrm{cm}) \\
\end{array}$ & $\begin{array}{c}\text { Material 0 } \\
\text { Absorption } \\
\text { Coefficient } \\
\sum_{a, 0}(1 / \mathrm{cm}) \\
\end{array}$ & $\begin{array}{c}\text { Material 1 } \\
\text { Scatter } \\
\text { Coefficient } \\
\Sigma_{\text {s.1 }}(1 / \mathrm{cm}) \\
\end{array}$ & $\begin{array}{c}\text { Material } 1 \\
\text { Absorption } \\
\text { Coefficient } \\
\sum_{\mathrm{a}, \mathrm{I}}(1 / \mathrm{cm}) \\
\end{array}$ \\
\hline 1 (abs + scat) & 10 & 10 & 0.01 & 0.01 \\
\hline 2 (pure absorbing) & 0 & 10 & 0 & 0.01 \\
\hline 3 (pure scattering) & 10 & 0 & 0.01 & 0 \\
\hline$\underline{4}(\mathrm{abs}+\mathrm{scat})$ & 10 & 10 & 0.1 & 0 \\
\hline 5 (abs + scat) & 10 & 10 & 1.0 & 0 \\
\hline 6 (abs + scat) & 10 & 10 & 2.5 & 0 \\
\hline
\end{tabular}

The physical dimensions of the bounding rectangle are the same for each problem. The width (W) and height $(\mathrm{H})$ are fixed at $20 \mathrm{~cm}$ and $50 \mathrm{~cm}$, respectively. The number of disks $(\mathrm{N})$ in each problem is also kept fixed at 50 . The radius of each disk is fixed at $0.5 \mathrm{~cm}$ for each case. The six cases represent a pure scattering case, a pure absorber case, and four cases of varying absorptive and scattering characteristics. For cases 1 through 3 , the magnitudes of the interaction coefficients are chosen to represent the commonly referenced case of a sparse 'black' material (M0) randomly mixed within an optically thin matrix material (M1). Reference (16) discusses this special case and the fact that the commonly applied 'atomic mix' model, wherein the stochastic mixture is converted to a homogeneous material of volume-fraction-averaged interaction coefficients, can't be expected to provide accurate results. In order to show that this is true, results are computed for all six cases using a simple homogenization of interaction coefficients.

Cases 4, 5, and 6 have the same interaction coefficients for material 0 as Case 1, but the absorption coefficient of material 1 is zero. For these cases, the scattering coefficient of material 1 is increased in order to study the effects on the accuracy of the Monte Carlo algorithms. 


\section{II.I Benchmark Method}

Figure 2 is a flow diagram that describes the Monte Carlo algorithm used to generate benchmark ensembleaveraged leakage results for the stochastic mixture problem. Results generated by this benchmark method provide "true" results that can be compared against results obtained by more approximate methods. Ensemble-averaged leakage results obtained by the Benchmark method are exact in the limit as the number of realizations approaches infinity, even if the number of histories per realization is only 1. For the Benchmark method, only 1 particle history is simulated per realization. This is necessary in order to provide a fair efficiency comparison to the 2 chord-length sampling algorithms which effectively sample a different realization for each particle.

A Monte Carlo code was written that follows the sequence of Figure 2 to calculate benchmark ensembleaveraged leakage results for the problems of interest. Homogeneous mixing of the disks for a given realization within the rectangle is achieved by randomly sampling the coordinates of each disk successively within the bounding area. Sampled disks are not permitted to overlap either on previously sampled disks or on the area boundaries. Once the realization is specified, then source particles are sampled from the source distribution for the problem and transport is simulated through the area until the particles either escape the boundary or are killed. Particle absorption is simulated implicitly using the weight reduction method rather than explicitly terminating the particle. When particle weight (W) drops below a weight cutoff limit, $\mathrm{C} 2$, a random number is sampled in the range $(0,1)$. If the random number is greater than $W$ divided by a second cutoff limit, $\mathrm{Cl}$, then the particle is terminated and a new history is begun. If the random number is less than $\mathrm{W} / \mathrm{Cl}$ then the particle weight is increased to $\mathrm{Cl}$ and the particle history is continued. This variance reduction method is common and is the same as used, for example, by the Los Alamos Monte Carlo particle transport code MCNP (17).

\section{II.III Chord-Length Sampling (CLS) Algorithm}

Figure 3 describes the CLS algorithm used in this work. Zimmerman $(14,15)$ first proposed the basic premise for this algorithm for tracking particles through a stochastic mixture. The important feature of this method is that it does not model the binary mixture explicitly. This eliminates the need to model a large number of complete physical realizations of the problem. Instead, the material identity for a given location is treated as a characteristic of the source particle. This capability could have significant advantages for a stochastic mixture containing thousands of randomly dispersed volumes. Instead of explicitly modeling all material volumes, all that is required are the material probabilities (volume fractions), and the chord-length distributions in each material.

Since the source particles are defined to enter the rectangle from the outside, the initial material identifier for the source particles is always chosen to be material 1. After the source particle's initial characteristics are sampled, three distance calculations are made. First the distance between the particle and the system boundary $D_{\mathrm{B}}$ is computed. The second calculation is the distance to collision $D_{\mathrm{C}}$. $D_{\mathrm{C}}$ is sampled using the 
current material's total interaction coefficient. These two distances are always computed in a Monte Carlo particle-tracking algorithm. The last distance calculation is of the distance between the particle and the material interface $D_{\mathrm{I}}$. In this algorithm, $D_{\mathrm{l}}$ is sampled from a chord-length distribution for the sampled material. The use of chord-length sampling reduces the calculation of the minimum distance to a disk to a simple sampling from a known probability distribution. The three distances are compared and the minimum distance dictates which event occurs.

If $D_{\mathrm{B}}$ is the minimum distance, then the particle escapes the system. The wall through which the particle escaped is noted and leakage is tallied. A new particle history is then begun. If $D_{\mathrm{C}}$ is the minimum, then a collision is sampled. The particle's position is updated and weight is reduced at each collision site based on the survival probability. As described in the previous section, particle rouletting is performed if the particle weight drops below a specified value. If the particle survives the collision (i.e., is not rouletted), then a new direction is sampled and the distance calculations are repeated. Finally, if the distance to material interface is the minimum distance, then the particle advances to the material interface and the material identifier is switched. After the particle's position and material are updated, then the distance calculations are repeated and the particle is tracked until it escapes or is rouletted.

The weakness of the CLS algorithm lies in the fact that the particle's past is ignored after each collision event. By allowing the particle's future to be independent of its past the CLS algorithm inherently assumes that the transport of a particle in a stochastic mixture is a Markovian process. In a Markovian transport process, the particle's prior interactions have no effect on the future interactions of the particle (5). (Note the distinction between the use of the terms 'Markovian Transport', which relates to a characteristic of the transport in a region, and 'Markovian Mixing Statistics', which specify an exponential distribution of chord lengths between material boundaries.)

Transport through a homogeneous material is Markovian. However, particle transport through a heterogeneous material such as a stochastic mixture is not a Markovian process because the distances to the surrounding material interfaces depend on the past trajectory of the particle. By re-sampling the distance to material interface following each collision, it is possible that the particle will be in a different material at one point in its history than it was in at the same position earlier in its history. Obviously, in general this is not physically possible. The only location where it would be possible for a particle to encounter either material at the same location is at the interface between the materials.

By allowing the material to be re-sampled at any position during a particle history, the CLS algorithm effectively makes the same assumption as the Levermore Approximation - that the average flux across material interfaces is equal to the average flux across the interior points. It must be noted here that for the problem defined, that of disks of uniform radius randomly mixed within a matrix, the mixing statistics are not strictly Markovian. The chord-length distribution in material 1 can be approximated by an exponential relation as we will show later, but the distribution in material ' 0 cannot. Because the Levermore 
-approximation is defined for a system with Markovian mixing statistics, the comparison made between it and the CLS Monte Carlo algorithm is only intended to be approximate.

For the case of purely absorbing materials for which exact chord length distributions are known, the CLS algorithm is expected to be exact. This statement can be easily understood when one considers that for the pure absorber, a particle can only move forward. Its past has no influence since there is no mechanism to scatter a particle back into a region through which it has already passed. Since the past is irrelevant to the particle in a purely absorbing medium, the transport is Markovian. Therefore, if the chord-length distributions are known exactly, then the CLS algorithm yields exact results. As scattering becomes more dominant in a given problem, the transport becomes more non-Markovian and the accuracy of the CLS algorithm will deteriorate.

\section{II.IV Limited Chord Length Sampling (LCLS) Algorithm}

In order to account for the non-Markovian nature of the transport process for stochastic mixtures, the effects of the particle's past have to be taken into account in the determination of the next event. The LCLS algorithm combines the chord-length sampling technique with limited explicit geometric representation of the stochastic mixture. This is done in order to reduce the errors that result from the assumption of Markovian transport implicit in the pure CLS algorithm. The algorithm takes advantage of the closed, simple geometry of the material 0 disks and treats these regions explicitly instead of using chord-length sampling. Chord length sampling is used for transport through material 1 and to specify the location of material 0 disks. Once a disk's location is specified, particle transport through the disk is done using traditional Monte Carlo. After the particle exits a disk, no memory of the disk is kept. Figure 4 shows a calculational schematic for the LCLS algorithm.

By modeling individual disks explicitly, this algorithm maintains a limited amount of memory regarding the particle's past. This 'partial memory' capability prevents a particle within an explicit material 0 disk from suddenly finding itself within material 1 without first escaping the disk. The LCLS algorithm reduces the assumption of pure Markovian transport made by the CLS algorithm. However, it is not entirely eliminated since each disk is discarded as soon as a particle exits it. Therefore upon backscatter from within material 1 the particle could 'see' a disk where there had previously been none, or vice versa. The concept of applying partial memory was also proposed by Zimmerman, and successfully applied in limited l-D slab problems. However, the implementation of partial memory for the binary stochastic mixture of this problem is unique.

The method for explicitly modeling a material 0 disk is now described. While in material $1, D_{\mathrm{B}}$ is calculated, $D_{\mathrm{C}}$ is sampled, and $D_{\mathrm{I}}$ is sampled from the material 1 chord-length probability distribution. If $D_{\mathrm{l}}$ is determined to be the minimum distance, then another sampling scheme is followed to determine the coordinates of the sampled disk. The location where the particle would cross into a material 0 disk is calculated based on the particle's position, it's direction, and $D_{\mathrm{l}}$. As shown in Figure 5, two random 
numbers are then sampled to determine the coordinates of the disk. The first random number $\xi 1$ samples a chord-length $\lambda_{0}$ from the probability distribution for a disk. The second random number determines the sense $( \pm)$ of the center of the disk relative to the chord.

After the coordinates of the disk have been sampled, the restriction that the disk may not overlap the boundary of the problem is tested. This is done by calculating the distances in the $\mathrm{x}$ and $\mathrm{y}$ directions from the disk coordinates to the four bounding walls of the problem. If any of the distances is less than the radius of the disk then the sampled disk overlaps one of the boundary walls and is not accepted. Once a disk is rejected for overlapping the boundary, the value of $D_{\mathrm{l}}$ is reset to a 'huge' value. The next event in the transport of the particle is based on the comparison of $D_{\mathrm{B}}$ and $D_{\mathrm{C}}$. If the disk is accepted, then the particle is moved forward to the intersection point and transport through the disk is performed.

\section{II.V Derivation of the Chord-Length Distributions}

The 2-dimensional binary stochastic mixture of interest consists of disks of material 0 randomly mixed within a second material, material 1 . Since there are two distinct material types in this mixture, there are two corresponding sets of chord-length distributions. These chord-length distributions describe the probability that a chord of length $\mathrm{dx}$ about $\mathrm{x}(\mathrm{p}(\mathrm{x}) \mathrm{dx})$ may be encountered as a ray makes a track though either material.

\section{II.V.I Material O (Disk) Chord-Length Distributions}

The chord-length distribution for the disk of radius $r$ will be derived first. In the case of a circle (disk) a chord is defined as a line segment whose endpoints both lie on the circle. The chord of a circle is also perpendicular to the radial line containing the midpoint of the chord. The derivation of the chord-length distributions for material 0 is fairly straightforward. We desire to determine the probability distribution of chords that are "randomly distributed" in a circle. The solution to this problem is dependent on exactly how the chords are randomly distributed. This phenomenon was first observed by Bertrand and is now known as Bertrand's Paradox (18). The different methods of defining random chords in a circle have been examined by Jaynes (19). Jaynes showed that the "natural" chord-length designation was that in which the polar coordinates of the chord midpoint $(\xi, \theta)$ are sampled randomly, where $\xi$ and $\theta$ are shown relative to the circle in Figure 6.

Looking at figure 6 it is apparent that any rotation of the circle does not change the length of the chord. Since the angle of rotation does not affect the chord distribution, we can then simplify the derivation of the chord-length distribution by assuming that the chord is horizontal, meaning that $\theta=0$. The chord length distribution then becomes that of a disk placed in a parallel beam.

To determine the transformation between the chord-length $\lambda$ and the random variable $\xi$ we equate

$$
p(\lambda) d \lambda={ }^{\prime} p(\xi) d \xi
$$


Rearrange equation 2 to separate the probability distribution for the chord-length and obtain .

$$
p(\lambda)=\frac{p(\xi)}{|d \lambda / d \xi|}
$$

The random variable $\xi$ is distributed uniformly from 0 to $\mathrm{r}$

$$
\xi=[0, r],
$$

where $r$ is the radius of the circle. Therefore the probability distribution for $\xi$ is simply

$$
p(\xi)=\frac{1}{r}
$$

The relationship between the sampled variable $\xi$ and the chord-length $\lambda$ is given from the equation for the circle.

$$
\lambda=2 \sqrt{r^{2}-\xi^{2}}
$$

Taking the derivative of this expression yields

$$
\left|\frac{d \lambda}{d \xi}\right|=\frac{2 \xi}{\sqrt{r^{2}-\xi^{2}}}
$$

Substitute equations 5 and 7 into 3 to yield the probability distribution function (PDF) for the chord-length.

$$
p(\lambda)=\frac{1 / r}{\frac{2 \xi}{\sqrt{r^{2}-\xi^{2}}}}=\frac{\lambda}{4 r \sqrt{r^{2}-\left(\frac{\lambda}{2}\right)^{2}}}
$$

The probability distribution is then integrated to find the cumulative probability distribution (CPD)

$$
P(\lambda)=\int_{b}^{\lambda} p(\lambda) d \lambda=1-\sqrt{1-\left(\frac{\lambda}{2 r}\right)^{2}}
$$

The CLS algorithm uses the chord-length distribution of equation 9 to sample all chords within the circles of the binary stochastic mixture. This is done out of necessity since the true distribution of chords from within the disk is not known. Equation 9 is only strictly valid for the first sampled chord after the particle enters the circle. After the particle penetrates the circle the distance to interface values would be smaller than the chord-lengths on average. Qualitatively, this implies that the use of the equation 9 CPD for sampling all chord-lengths within a circle may underpredict the probability of a particle to escape a circle. This in turn may cause an underprediction of the overall leakage probabilities for the problem. However, for highly absorbing material 0 regions it is expected that this approximation will not have significant impact on the results. The LCLS algorithm only uses the chord-length distribution of equation 9 to sample the first chord. This chord is then used to fix the coordinates of an explicit disk. 


\section{II.V.II Material 1 Chord length distributions}

It does not appear feasible to analytically derive the chord-length distribution for chords between the disks. Pomraning (2) examined this problem and showed that, by assuming fixed circle chord lengths equal to the average chord length, in the limit for very large systems with homogeneous mixing, the PDF for chords in material 1 reduces to a Markovian model. We assume that the Markovian model is an acceptable approximation for chords in material 1 for this problem. This means that the material 1 chords fall in an exponential distribution of the form

$$
p(\lambda)=\frac{1}{\bar{\lambda}_{1}} \cdot e^{-\lambda \cdot \frac{1}{\bar{\lambda}_{1}}}
$$

Where $\lambda$ is the chord-length and $\bar{\lambda}_{1}$ is the mean chord-length through material 1 . The cumulative probability distribution is obtained by integrating equation 10 .

$$
P(\lambda)=\int_{0}^{\lambda} p(\lambda) d x=1-e^{-\frac{\lambda}{\bar{\lambda}_{1}}}
$$

Equation 11 is a simple expression that can be used to sample chords through material 1. A key difference between this distribution and the distribution for material 0 is that the mean chord-length for material 1 must be known or approximated in order to use the distribution.

The value of $\bar{\lambda}_{1}$ was determined empirically by using a Monte Carlo sampling method. A Monte Carlo routine was written to randomly place $\mathrm{N}$ circles of radius $\mathrm{r}$ within a rectangle of thickness $\mathrm{L}$ and Height $\mathrm{H}$, where the values of these parameters were the same as defined for the six cases to be examined. Again, disks were not permitted to overlap any other disks or any of the boundaries of the rectangle. If no circles fell along the horizontal line of length $\mathrm{L}$ in the center of the rectangle, then a positive score was tallied. This process was repeated many times and the probability of 0 circles falling on the line was calculated. The probability that no circles fall on the horizontal line is denoted by $\mathrm{P}(0)$. This probability can also be interpreted as the probability of chord-lengths greater than length $L$ in material 1 . From this interpretation, an expression for $\mathrm{P}(0)$ can be obtained by integrating equation 11 over the appropriate limits.

$$
P(0)=P\left(\lambda_{1}>(L-2 r)\right)=\int_{L-2 r}^{\infty}\left(\frac{1}{\bar{\lambda}_{1}} \cdot e^{-\lambda_{1} \cdot \frac{1}{\bar{\lambda}_{1}}}\right) d x
$$

Note that the limits of this expression take into account the diameter (2r) of the disks. This is required since over the horizontal distance $L$, and allowing no overlap of disks with the boundaries, the permissible range of the disk coordinates spans a distance of L-2r. Once $\mathrm{P}(0)$ is known empirically, then the average chordlength can be determined by evaluating the integral in equation. 14 and solving for $\bar{\lambda}_{1}$.

$$
\overline{\lambda_{1}}=\frac{2 R-L}{\ln (P(0))}
$$


For given values of $\mathrm{N}, \mathrm{r}, \mathrm{L}$, and $\mathrm{H}$ stated for this problem, $\mathrm{P}(0)$ was determined to be 0.3393 based on 2

million realizations of the mixture. Based on this value, $\bar{\lambda}_{1}$ was then calculated using equation 15 and determined to be $17.58 \mathrm{~cm}$.

\section{Standard Deviation of Monte Carlo Results}

Solution of the Stochastic Transport equation with traditional Monte Carlo techniques is accomplished by simulating the transport of a given number of particles through a unique geometrical realization of the stochastic mixture. For each physical representation, a number of particle histories (i) is simulated and results are tallied for the quantities of interest (for example: flux, reaction rate, leakage, etc). The simulation is then repeated for large number of realizations ( $j$ ). The ensemble-average of all the individual results is then estimated from the population of results that have been gathered. For the CLS and LCLS algorithms the number of histories per realization is effectively one. In order to provide one-to-one efficiency comparisons against the CLS and LCLS algorithms, Benchmark results are also computed using only one history per realization. For allthree algorithms, the ensemble-averaged result is estimated as

$$
\overline{\bar{x}}_{j}=\frac{\sum_{j=1}^{N_{\text {ITER }}} x_{j}}{N_{\text {ITER }}},
$$

where $\mathrm{x}_{\mathrm{i}, \mathrm{j}}$ is the tally result for realization $\mathrm{j}$ and $\mathrm{N}_{\text {ITER }}$ is the number of realizations.

There are two useful statistical quantities associated with this ensemble-averaged result. The first is the standard deviation of the population of results from $\mathrm{N}_{\text {ITER }}$ realizations. This parameter describes the variability in results between successive realizations due both to the stochastic method of particle transport and the stochastic nature of the geometry. The standard deviation of the population of $\mathrm{N}_{\text {ITER }}$ results is estimated using the expression

$$
S_{x_{j}}=\left(\frac{\sum_{j=1}^{N_{\text {iter }}} x_{j}^{2}}{N_{\text {iter }}}-\overline{\bar{x}}_{j}^{2}\right)^{\frac{1}{2}} .
$$

The second statistical quantity is the estimated standard deviation of the ensemble-averaged result itself. This quantity is a measure of the accuracy of the ensemble-averaged quantity relative to the "true" quantity, which is unknown. The estimated standard deviation of the ensemble-averaged result is equal to

$$
S_{\vec{x}_{j}}^{2}=\frac{S_{x_{j}}^{2}}{N_{\text {ITER }}}
$$




\section{IV.I Results}

Table 2 shows the leakage results through all four walls for the six cases. Leakage results were calculated using the Benchmark algorithm and the two chord-length sampling algorithms. For comparison purposes, results were also calculated by homogenizing the interaction cross sections of both materials into a single averaged material. This was done to verify that statement made earlier that a simple homogenization of these problems could not produce accurate results. Included in parentheses are the estimated errors of the ensemble-averaged results, calculated using Equation 18.

Table 2: $\quad$ Calculated Leakages for Six Cases (Parentheses contain 1 Standard Deviation Estimate).

\begin{tabular}{|c|c|c|c|c|}
\hline $\begin{array}{l}\text { Solution } \\
\text { Method }\end{array}$ & $\begin{array}{c}\text { Reflection } \\
\text { through Left Wall } \\
\text { (Tl) }\end{array}$ & $\begin{array}{l}\text { Leakage through } \\
\text { Right Walt } \\
(T 2)\end{array}$ & $\begin{array}{c}\text { Leakage through } \\
\text { Top Wall } \\
\text { (T3) }\end{array}$ & $\begin{array}{c}\text { Leakage through } \\
\text { Bottom Wall } \\
\text { (T3) }\end{array}$ \\
\hline \multicolumn{5}{|c|}{ Case \# 1 (abs + scat): $\Sigma_{a, 0}=10, \Sigma_{s, 0}=10, \Sigma_{a, 1}=0.01, \Sigma_{s, 1}=0.01$} \\
\hline Benchmark & $0.0565(0.0006)$ & $0.249(0.002)$ & $0.0057(0.0002)$ & $0.0058(0.0002)$ \\
\hline CLS & $0.0231(0.0005)$ & $0.238(0.002)$ & $0.0030(0.0002)$ & $0.0031(0.0002)$ \\
\hline LCLS & $0.0582(0.0008)$ & $0.247(0.002)$ & $0.0059(0.0002)$ & $0.0057(0.0002)$ \\
\hline Atomic Mix & $0.1148(8.05 \mathrm{E}-5)$ & 2.1E-7 (6E-8) & $6.3 \mathrm{E}-9(3 \mathrm{E}-9)$ & $3.3 \mathrm{E}-9(1.5 \mathrm{E}-9)$ \\
\hline \multicolumn{5}{|c|}{ Case \# 2 (absorbing): $\Sigma_{8,0}=10, \Sigma_{s, 0}=0.0, \Sigma_{\mathrm{s}, 1}=0.01, \Sigma_{s, 1}=0.0$} \\
\hline Benchmark & 0.0 & $0.283(0.002)$ & 0.0 & 0.0 \\
\hline CLS & 0.0 & $0.282(0.002)$ & 0.0 & 0.0 \\
\hline LCLS & 0.0 & $0.281(0.002)$ & 0.0 & 0.0 \\
\hline Atomic Mix & 0.0 & $3.23 \mathrm{E}-4(1.04 \mathrm{E}-5)$ & 0.0 & 0.0 \\
\hline \multicolumn{5}{|c|}{ Case \# 3 (scattering): $\Sigma_{a, 0}=0.0, \Sigma_{s, 0}=10, \Sigma_{a, 1}=0.0, \Sigma_{s, 1}=0.01$} \\
\hline Benchmark & $0.395(0.002)$ & $0.460(0.002)$ & $0.073(0.001)$ & $0.073(0.001)$ \\
\hline CLS & $0.324(0.002)$ & $0.503(0.002)$ & $0.086(0.001)$ & $0.088(0.001)$ \\
\hline LCLS & $0.388(0.002)$ & $0.464(0.002)$ & $0.074(0.001)$ & $0.074(0.001)$ \\
\hline Atomic Mix & $0.773(0.0002)$ & $0.132(0.0002)$ & $0.047(0.0001)$ & $0.047(0.0003)$ \\
\hline \multicolumn{5}{|c|}{ Case \# 4 (abs + scat) $: \Sigma_{a, 0}=10, \Sigma_{s, 0}=10, \Sigma_{g, 2}=0.0, \Sigma_{s, 1}=0.1$} \\
\hline Benchmark & $0.227(0.002)$ & $0.0999(0.001)$ & $0.0134(0.0005)$ & $0.0125(.0005)$ \\
\hline CLS & $0.184(0.001)$ & $0.092(0.001)$ & $0.0102(0.0003)$ & $0.0102(0.0003)$ \\
\hline LCLS & $0.222(0.001)$ & $0.100(0.001)$ & $0.0128(0.0004)$ & $0.0121(0.0004)$ \\
\hline Atomic Mix & $0.137(0.00008)$ & $1.6 \mathrm{E}-7(4.8 \mathrm{E}-8)$ & $3.5 \mathrm{E}-9(1.5 \mathrm{E}-9)$ & $7.6 \mathrm{E}-10(5.7 \mathrm{E}-10)$ \\
\hline \multicolumn{5}{|c|}{ Case \# 5 (abs + scat) $): \Sigma_{a, 0}=10, \Sigma_{s, 0}=10, \Sigma_{a, 1}=0.0, \Sigma_{s, 1}=1.0$} \\
\hline Benchmark & $0.635(0.002)$ & $0.0010(0.0001)$ & $0.00036(0.00008)$ & $0.00034(0.00007)$ \\
\hline CLS & $0.568(0.002)$ & $0.0003(0.0001)$ & $0.00018(0.00004)$ & $0.00008(0.00003)$ \\
\hline
\end{tabular}




\begin{tabular}{|r|l|l|l|l|}
\hline $\begin{array}{r}\text { Solution } \\
\text { Method }\end{array}$ & $\begin{array}{c}\text { Reflection } \\
\text { through Left Wall } \\
(\mathrm{T} 1)\end{array}$ & $\begin{array}{c}\text { Leakage through } \\
\text { Right Wall } \\
(\mathrm{T} 2)\end{array}$ & $\begin{array}{c}\text { Leakage through } \\
\text { Top Wall } \\
(\mathrm{T} 3)\end{array}$ & $\begin{array}{c}\text { Leakage through } \\
\text { Bottom Wall } \\
(\mathrm{T} 3)\end{array}$ \\
\hline LCLS & $0.594(0.002)$ & $0.0004(0.0001)$ & $0.00020(0.00004)$ & $0.00020(0.00004)$ \\
\hline Atomic Mix & $0.261(0.0001)$ & $*$ & $*$ & $*$ \\
\hline \multicolumn{5}{|c|}{ Case \# 6 (abs + scat) $: \Sigma_{\mathrm{a}, 0}=10, \Sigma_{\mathrm{s}, 0}=10, \Sigma_{\mathrm{a} .1}=0.0, \Sigma_{s, 1}=\mathbf{2 . 5}$} \\
\hline Benchmark & $0.786(0.002)$ & $0.00017(0.00006)$ & $0.00003(0.00002)$ & $*$ \\
\hline CLS & $0.707(0.003)$ & $*$ & $*$ & $*$ \\
\hline LCLS & $0.729(0.003)$ & $4 \mathrm{e}-6(\mathrm{le}-6)$ & $2 \mathrm{e}-6(7 \mathrm{e}-7)$ & $2.8 \mathrm{e}-6(8 \mathrm{e}-7)$ \\
\hline Atomic Mix & $0.376(0.0001)$ & $*$ & $*$ & $*$ \\
\hline * No tallies were scored across the wall for this case & \\
\hline
\end{tabular}

The value of $P(0)$ used in the two chord-length sampling algorithms was 0.3393 . This value was obtained by simulating 2 million realizations of the stochastic mixture and tallying the number of times a realization was created in which no disks overlapped the $\mathrm{x}$ axis as shown in Figure 1. The time required to empirically determine $P(0)$ is not included in the timing studies. It is expected that for future studies, an analytical approximation of $\mathrm{P}(0)$ will be established as a function of disk radius, the number of disks, and the region height and width $(\mathrm{H}, \mathrm{W})$. Table 3 shows the relative timing results for each algorithm and for each case. Timing results are presented as relative to the time required to determine the Benchmark result.

Table 3: $\quad$ Relative Timing Comparison between the Benchmark and the Two Chord Length Sampling Algorithms for Six Cases

\begin{tabular}{|c|c|c|c|}
\hline Solution Method & Relative Timing & Solution Method & Relative Timing \\
\hline \multicolumn{2}{|c|}{ Case \# 1 (abs + scat) } & \multicolumn{2}{|c|}{ Case \# 4 (abs + scat) } \\
\hline Benchmark & 1 & Benchmark & 1 \\
\hline CLS & 0.1368 & CLS & 0.156 \\
\hline LCLS & 0.1042 & LCLS & 0.132 \\
\hline \multicolumn{2}{|c|}{ Case \# 2 (pure absorbing) } & \multicolumn{2}{|c|}{ Case \# 5 (abs + scat) } \\
\hline Benchmark & 1 & Benchmark & 1 \\
\hline CLS & 0.0520 & CLS & 0.155 \\
\hline LCLS & 0.0446 & LCLS & 0.2 \\
\hline \multicolumn{2}{|c|}{ Case \# 3 (pure scattering) } & \multicolumn{2}{|c|}{ Case \# 6 (abs + scat) } \\
\hline Benchmark & 1 & Benchmark & 1 \\
\hline CLS & 1.887 & CLS & 0.157 \\
\hline LCLS & 0.1855 & LCLS & 0.223 \\
\hline
\end{tabular}




\section{Conclusions}

The results of Table 2 show that both algorithms provide exact results for the purely absorbing case but provide different levels of accuracy as scattering is introduced in the problems. For the purely absorbing case, both algorithms are within the statistics of the benchmark method. This case is a Markovian transport problem and assuming that the chord-length distributions are correctly represented, than the solution for such a case is expected to be exact.

Once scattering is introduced in a problem, neither algorithm is exact since neither keeps track of prior events and conditions. Furthermore, for the CLS algorithm, it has been stated that for the scattering cases the chord-length distribution within a disk becomes more approximate. This is because the derived chordlength distribution is only strictly applicable for chords spanning the entire disk. For a particle within a disk the distance to interface is only approximately represented by this distribution. Therefore we have two reasons to expect that the accuracy of the CLS algorithm will decrease as scattering in the system increases. For the LCLS algorithm, there is no chord-length distribution needed for the disks. Therefore, while LCLS is vulnerable to error due to it's assumption of Markovian transport, it does not contain the source of error due to the use of an approximate disk chord-length distribution.

For Case 1, the CLS results for leakage through the right wall (T2) are within 5\% of the Benchmark. This good agreement is expected since the predominant contributor to the leakage through wall 2 is the uncollided particle fraction. For the leakage fractions that are more dependent on particle scattering, CLS results are significantly different than the Benchmark. For Case 1, CLS underpredicts the particle reflection back out of the left wall (T1) by $59 \%$. For this case, CLS also underpredicts the leakage out of the top and bottom walls by $47 \%$ and $46 \%$, respectively. The CLS algorithm also shows significant differences as compared to the Benchmark results for the purely scattering problem (Case 3). For case 3, predicted leakage through the right wall has the smallest error and is $9 \%$ higher than the Benchmark result. Reflection back out the left side is $18 \%$ lower than the Benchmark, while leakage through the top and bottom walls was approximately $19 \%$ high for the CLS algorithm relative to the Benchmark.

The fact that CLS overpredicts leakage and underpredicts reflection for the purely scattering case is due primarily to the chord-length distribution that was used for chords in a disk. In the Benchmark algorithm as well as LCLS, a given disk is fixed in space. If that disk has a high interaction coefficient it is likely that a particle will only penetrate the disk a small distance before having a collision. At that collision point, the distance forward a particle would have to go to escape the disk is most likely greater than the distance backward the particle would have to go to escape the disk. In CLS, once a particle collides within a disk, the distance to escape is always sampled from the same distribution. The probability of a particle escaping a disk after colliding is a function only of the mean chord-length and is independent of whether the scatter was forward or backward. Qualitatively therefore, the effect relative to the Benchmark algorithm or LCLS is that the probability of a particle escaping a disk after a backscatter goes down, while the probability of 
escaping a disk after a forward scatter goes up. This effect is not observed in Case 1 due to the high . absorption coefficient within the disks.

The problem described above with the chord-length distribution used for chords in a disk also has a very strong negative impact on the computational efficiency of CLS for the purely scattering case. Table 3 shows that for Case 3 CLS runs $88 \%$ slower than the benchmark method. This is due to the fact that the chord-length distribution has a trapping effect on particles that enter a disk. Because there is no absorption in the disks for this case, particles have no choice but to scatter within a disk until they escape. Because the chord-length distribution makes escape more difficult, the result is an increase in the time spent transporting the particles. The reason why this increase in time is not as pronounced in CLS for the other cases is due to the presence of high absorption in material 0 . Once absorption is introduced, the trapping effect of the chord-length distribution is not as noticeable since particles are quickly absorbed in the disks.

LCLS substantially reduces the errors obtained using CLS by eliminating the need to sample chord-lengths within the disks. For the first three cases, LCLS results for the cases examined are within a range of $\pm 3 \%$ in general relative to the Benchmark results. LCLS results for Cases 4, 5, and 6 show that accuracy degrades as scattering is increased. Results for Case 6, which has the highest scattering coefficient for material 1 , show a complete breakdown in accuracy for all results except for the reflected fraction, which is still within $10 \%$ of the benchmark.

LCLS also shows considerable computational savings relative to the Benchmark algorithm. Time savings factors from LCLS, compared to the Benchmark, of 9.6, 22.4, and 5.4 for cases 1 through 3, respectively. Time savings decrease as scattering increases. This is due to the fact that as the scattering probability increases, the time required for particle transport goes up. In the Benchmark algorithm, as the time requirement for particle transport goes up, the time requirement to sample each realization relative to the total job time goes down. 


\section{References}

1) G.L. Stephens, "The Parameterization of Radiation for Numerical Weather Prediction and Climate Models," Monthly Weather Review, 112, 826, 1984

2) B. Su, G.C. Pomraning, "A Stochastic Description of a Broken Cloud Field", Transactions of the American Meteorological Society, pp. 1969-1977, July 1994

3) M. Noskova, A. Valentyuk, "Stochastic Laser Beam Transfer Through a Binary Markovian Mixture",Applied Optics, Vol. 36, No 30, October 1997

4) J.K. Shultis, R.E. Faw, "Radiation Shielding", Prentis Hall, 1996

5) O. Haran, D. Shvarts, R. Thieberger, "Transport in Two Dimensional Scattering Media: Simulations and Models", Physical Review E, Vol. 61, Number 6, pp. 6183-6189, June 2000

6) G.C. Pomraning, H. Frisch, P.F. Zweifel, "An Exact Analytical Solution of a Radiative Transfer Problem in a Binary Mixture", Journal of Quantitative Spectroscopic Radiation Transfer, Vol 43, pp. 271-284, 1990

7) G.C. Pomraning, "The Variance in Stochastic Transport Problems with Markovian Mixing", JQSRT, Vol 56, pp. 629-646, 1996

8) M.L. Adams, E.W. Larsen, and G.C. Pomraning, "Benchmark Results for Particle Transport in a Binary Markov Statistical Medium,", JQSRT, 42, pp. 253, 1989

9) G.C. Pomraning, B. Su, "Modification to a Previous Higher Order Model for Particle Transport in Binary Stochastic Media", JQSRT, 54, pp. 779-801, 1995

10) B.S. Ching and T.S. Palmer, "Analysis of Iterative Schemes for Binary Stochastic Mixture Transport Equations", Submitted to the American Nuclear Society Summer Conference, June 2000, San Diego, CA

11) M.M.R. Williams, "Neutron Transport in Spatially Random Media: An Assessment of the Accuracy of First Order Smoothing", Nuclear Science and Engineering: 135, 123-140, 2000

12) M.M.R. Williams, "Some Aspects of Neutron Transport in Spatially Random Media", Nuclear Science and Engineering: 136,34-58,2000

13) F. Malvagi, G.C. Pomraning, "A Comparison of Models for Particle Transport Through Stochastic Mixtures, "Nuclear Science and Engineering: 111, 215-228

14) G.B. Zimmerman, M.L. Adams, "Algorithms for Monte Carlo Particle Transport in Binary Statistical Mixtures", Transactions of the American Nuclear Society, pp. 286-294, 1991

15) G.B. Zimmerman, "Recent Developments in Monte Carlo Techniques", UCRL-JC-105616, Lawrence Livermore National Lab, 1990

16) G.C. Pomraning, "Linear Kinetic Theory and Particle Transport in Stochastic Mixtures", 1991

17) LANL XTM Monte Carlo Team, "MCNP - A General Monte Carlo N-Particle Transport Code. Version 4B", Transport Methods Group, Los Alamos National Laboratory, 1997

18) J. Bertrand, Calcul des Probabilites, Paris: Gauthier-Vallars, 1889

19) E.T. Jaynes, "The Well Posed Problem", Papers on Probability, Statistics, and Statistical Physics, R.D. Rosencrantz, ed. ,Dordrecht: D. Reidel, 1983, pp. 133-148 


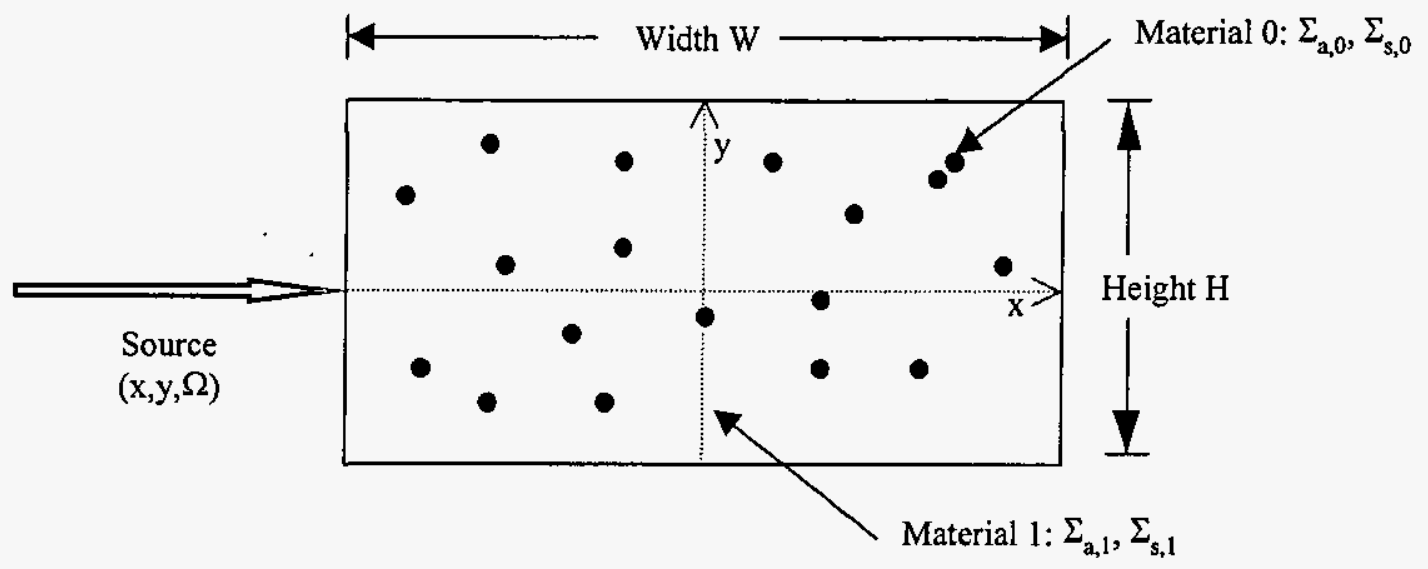

Figure 1: Binary stochastic mixture: disks embedded in a matrix $\Sigma_{\mathrm{a}, \mathrm{i}}$ Absorption coefficient of material i [ $\left.\mathrm{cm}^{-1}\right]$

$\Sigma_{\mathrm{s}, \mathrm{i}}$ Scattering coefficient of material $\mathrm{i}\left[\mathrm{cm}^{-1}\right]$ 


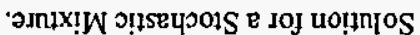

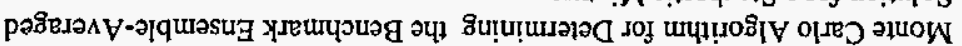

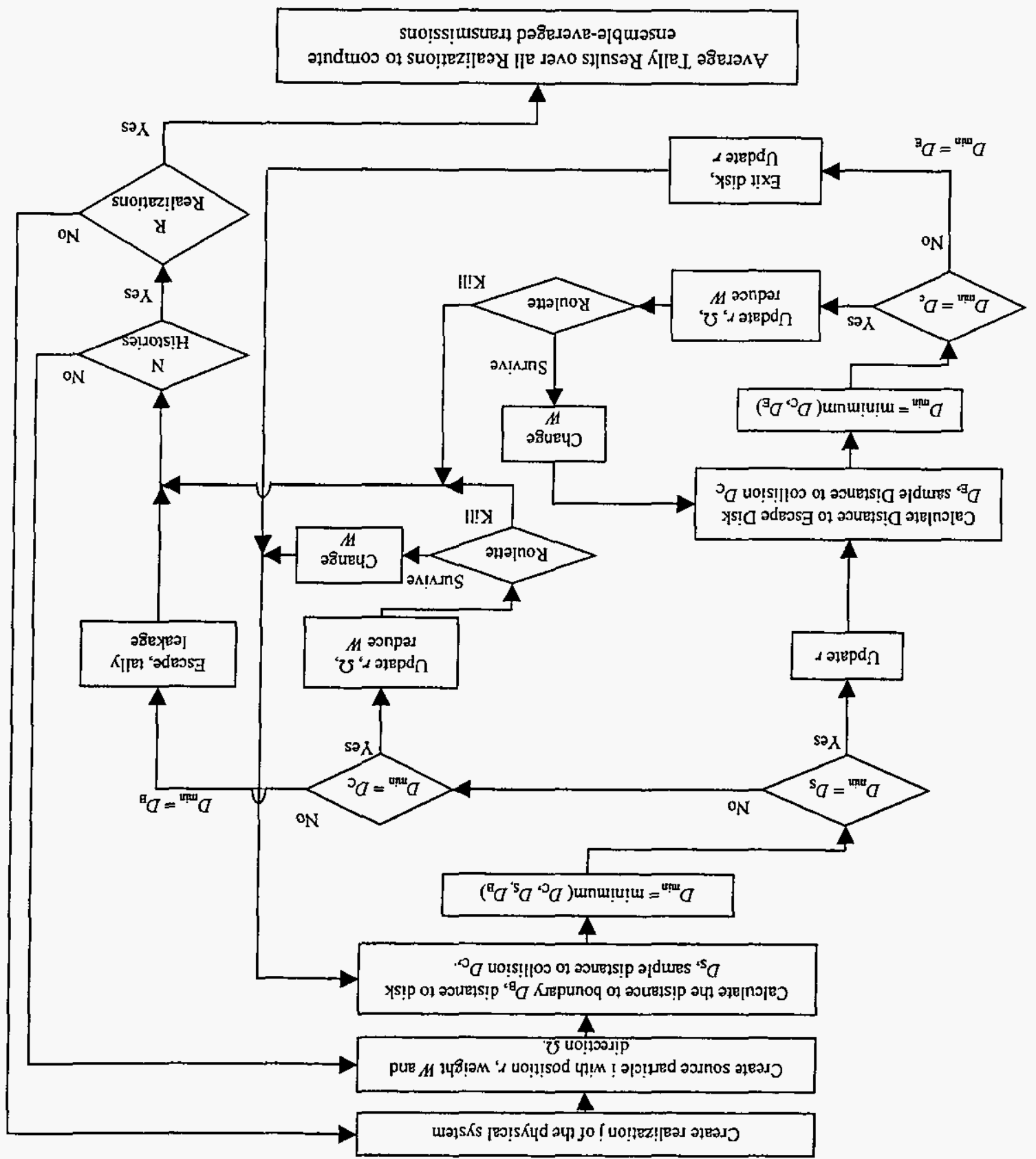




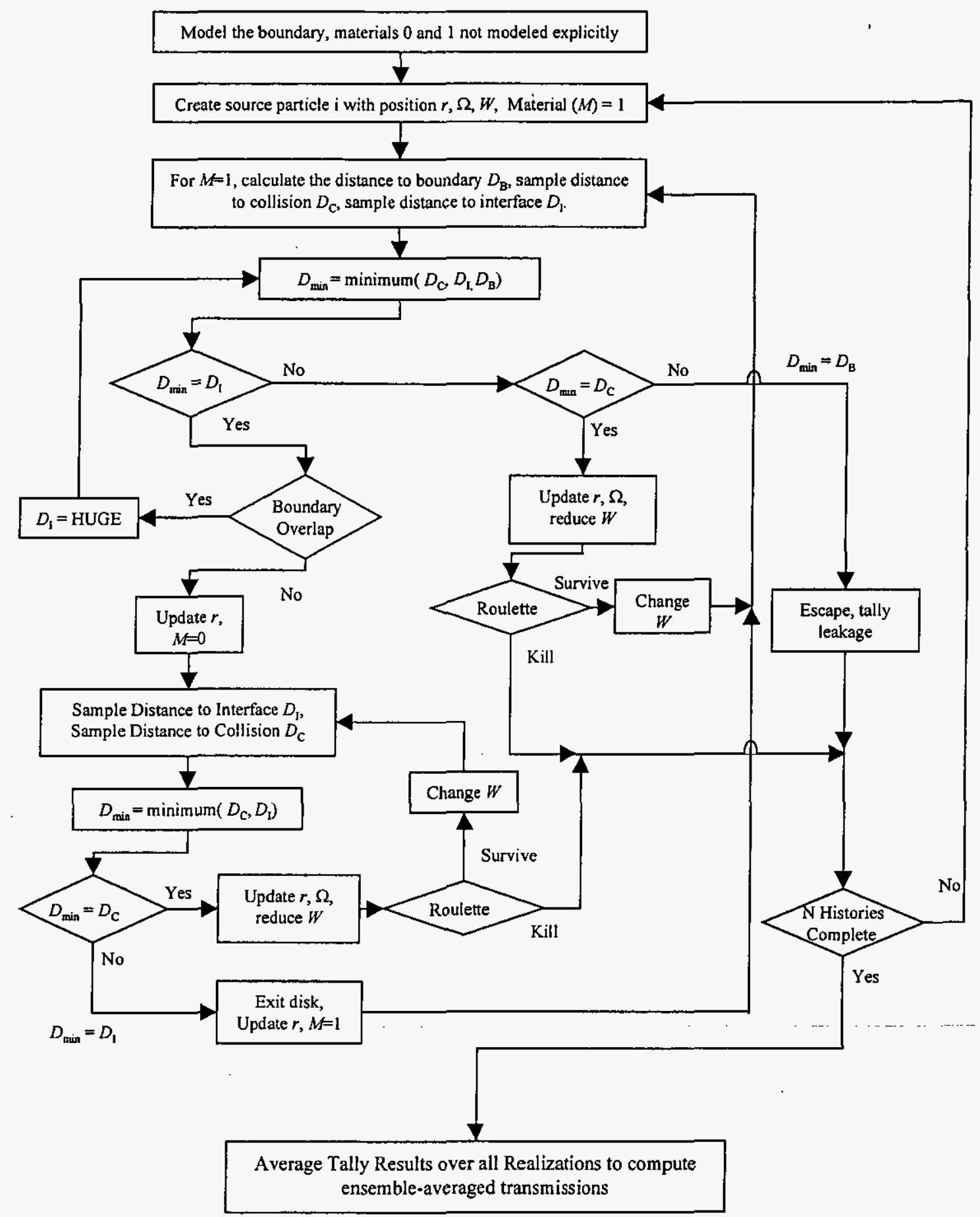

Figure 3: Chord Length Sampling (CLS) Method 


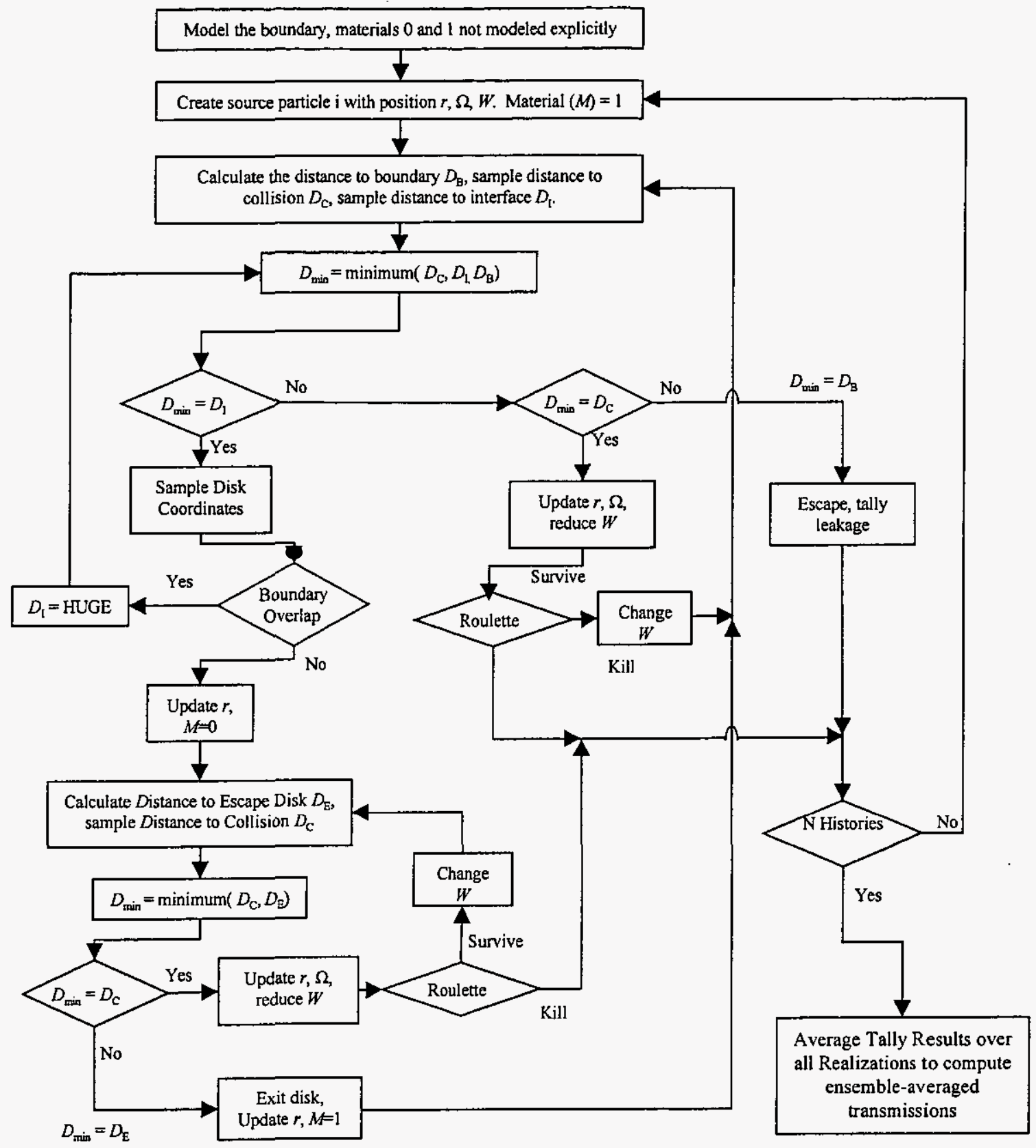

Figure 4: Limited Chord Length Sampling Method (LCLS) 


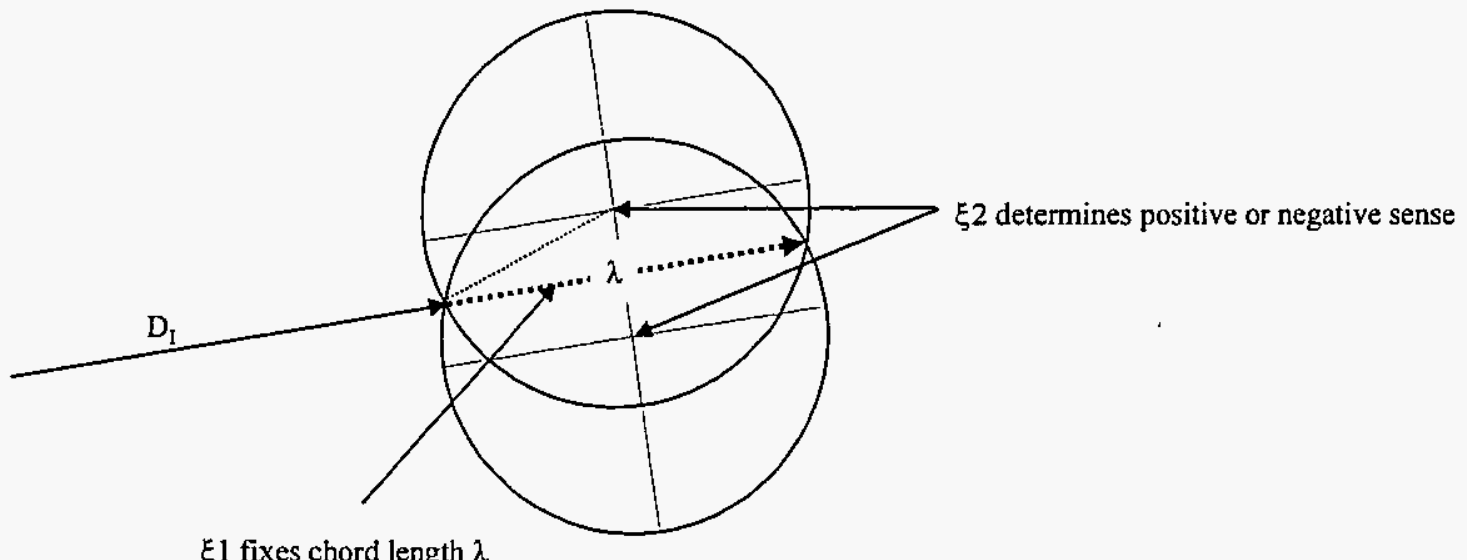

$\xi 1$ fixes chord length $\lambda$

Figure 5: Determination of Disk Coordinates for the LCLS Algorithm

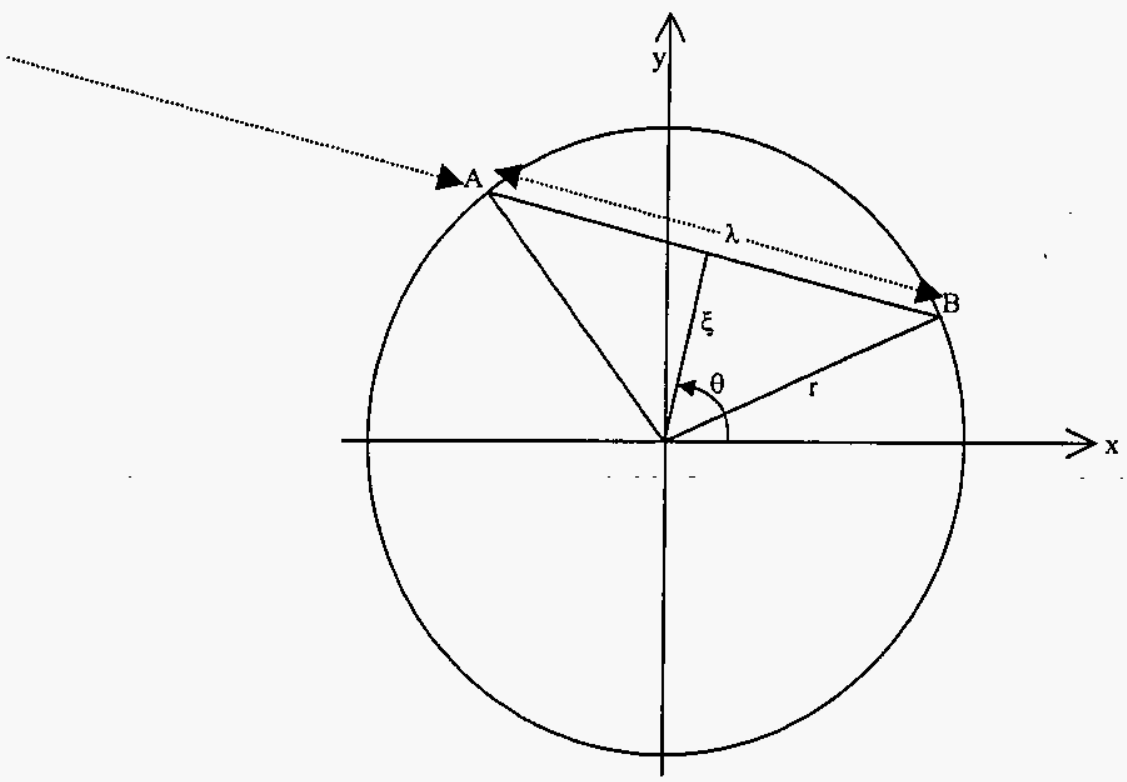

Figure 6: "Random" Chord in a Circle 